\title{
Development of lock-in based overtone Modulated MARY spectroscopy for detection of weak magnetic field effects ${ }^{\text {a) }}$
}

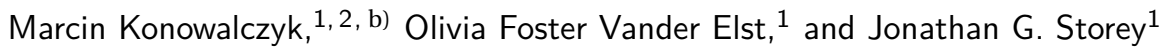 \\ ${ }^{1)}$ Department of Chemistry, University of Oxford, Inorganic Chemistry Laboratory, Oxford OX1 3QR, U.K. \\ 2) Department of Chemistry, University of Oxford, Physical and Theoretical Chemistry Laboratory, Oxford OX1 3QZ, U.K.
}

(Dated: December 6, 2020)

\begin{abstract}
Modulated magnetically altered reaction yield (ModMARY) spectroscopy is a derivative variant of fluorescence detected magnetic field effect measurement, where the applied magnetic field has both a constant and a modulated component. As in many derivative spectroscopy techniques, the signal to noise ratio scales with the magnitude of the modulation. High modulation amplitudes, however, distort the signal and can obscure small features of the measured spectrum. In order to detect weak magnetic field effects (including the low field effect) a balance of the two has to be found. In this work we look in depth at the origin of the distortion of the MARY signal by field modulation. We then present an overtone detection scheme, as well as a data analysis method which allows for correct fitting of both harmonic and overtone signals of the modulation broadened MARY data. This allows us to robustly reconstruct the underlying MARY curve at different modulation depths. To illustrate the usefulness of the technique, we show measurements and analysis of a well known magnetosensitive system of pyrene / 1,3-dicyanobenzene (Py/DCB). The measurements of first $\left(h_{1}\right)$ and second $\left(h_{2}\right)$ harmonic spectra are performed at different modulation depths for both natural isotopic abundance ( $\left.\mathrm{PyH}_{10}\right)$, and perdeuterated (PyD10) pyrene samples.
\end{abstract}

Keywords: Magnetic field effect; lock-in amplification; quadrature; derivative; overtone; spectroscopy; convolution; modelling; nonlinear fitting; bootstrap; exciplex system; radical pair mechanism; magnetoreception

\section{INTRODUCTION}

Many chemical systems exhibit sensitivity to comparatively weak magnetic fields ${ }^{2,3}$ by a variety of mechanisms. ${ }^{4-6}$ The most prevalent of mechanisms giving rise to a magnetic field effect (MFE) is the radical pair mechanism. ${ }^{5}$ This mechanism typically relies on photoinduced creation of a radical pair (RP) and the magnetically alerted reaction yield (MARY) of its products. Briefly, the singlet RP can recombine to the ground state through a non-radiative pathway, return to a higher energy singlet state and fluoresce, or interconvert with the triplet RP state. The rate of this interconversion is altered by the magnetic field, as the triplet states of the RP possess a magnetic moment which can interact with an externally applied magnetic field (the Zeeman effect). The triplet RP has a more limited range of spin-allowed reactions. Notably, the nonradiative recombination of the triplet $\mathrm{RP}$ to the ground state is usually spin-forbidden. Hence, the singlet-triplet interconversion alters the quantum yield of different products downstream from the RP. The resulting magnetically-induced changes in the population of these products accumulate over the course of multiple turnovers of the photocycle. This alters the fraction of species in the ground state and consequently gives rise to a magneticfield induced change in the observed fluorescence intensity.

\footnotetext{
a) Electronic Supplementary Information (ESI) available from Oxford University Research Archive (ORA) at ora .ox. ac. uk $^{1}$

${ }^{b)}$ Corresponding author. Please contact marcin . konow@lczyk. xyz
}

Of particular interest for avian magnetoreception research ${ }^{7}$ is the low-field effect (LFE). The LFE is a high contrast in the magnetochemistry between zero and low field $(\sim 1 \mathrm{G})$ conditions. ${ }^{8}$ There exist additional coherences between the interconverting spin states of the radical pairs which are unlocked at low, as opposed to zero external magnetic field. ${ }^{9}$ This usually leads to a magnetic response of the chemical system which, at low fields, is inverted with respect to that at higher fields. The plot of a magnetically altered property of a sample (e.g. the fluorescence intensity) as a function of applied magnetic field $b$ is referred to as a MARY curve. An example of a MARY curve can be seen in Figs. $1 \mathrm{~b}$ and 5 . The LFE feature can be seen on the insert of Fig. 5 .

Modulated MARY (ModMARY) spectroscopy is a variant of fluorescence spectroscopy used for detection of magnetic field effects where the applied magnetic-field has both a static and a modulated component. A sample of interest is placed in an oscillating magnetic field (frequency $f_{m}$, amplitude $b_{m}$ ) and is continuously excited with an appropriate wavelength light in order to establish an oscillatory steady state fluorescence (see Fig. 1a). The magnetosensitive components of the fluorescence will then oscillate at the frequency (and overtones) of $f_{m}$. The amplitude of this oscillation is measured as a function of a static offset field $\left(b_{o}\right)$. This could be done simply by notch filtering of a Fourier transform of the digitised signal. However, much narrower bandwidths, and thus better noise rejection, are usually achieved with lock-in detection. ${ }^{10-13}$ The lock-in amplifiers (LIAs) are, therefore, the most common way of demodulating the fluorescence signal in ModMARY experiments. ${ }^{14-17}$ 
The $f_{m}$ component of the total applied magnetic field $(b)$ is usually small and thus the signal detected is proportional to the derivative of the measured MARY curve (see Fig. 1b). However, in order to achieve the high signal to noise ratio required to measure small field effects, a much higher modulation amplitude is often necessary, which can in turn distort the signal.

ModMARY spectroscopy is most commonly employed for measurements of fluorescence MFEs on exciplex ${ }^{15}$ and solid-state systems. ${ }^{18}$ Due to the field-modulation employed, the signal detected is related to the derivative of the MARY curve. One of the utilities of ModMARY is therefore its ability to determine the width of the MARY curve with high accuracy and precision. This has been widely employed in studies of self-exchange in magnetosensitive systems based in both homogenous ${ }^{19}$ and micellar solutions, ${ }^{16}$ and to investigate RP-sovent interactions. ${ }^{17}$ Since ModMARY can easily determine the presence of a low-field effect it has also been used in exploratory studies of new polyarenebased compass chemical systems, ${ }^{18}$ and in experimental investigations of the effects of the hyperfine coupling on the LFE. ${ }^{20}$

There are other, related, techniques which make use of the field-modulation and derivative detection. In recent years, field-modulation technique has been combined with laser scanning to yield both fluorescence $\mathrm{e}^{21}$ and absorptionbased ModMARY imaging 22,23 (referred to as magnetofluorescence imaging (MFI) and magnetic intensity modulation (MIM) imaging respectively). The general technique of stimulus-modulation can be seen in many fields of spectroscopy, most notably optical spectroscopy, for example in frequency (wavelength) modulation using tunable light sources, cavity spectroscopy and electron paramagnetic resonance (EPR).

The simplest method of analysing a derivative signal is to ignore the influence of the modulation on the signal shape and treat it as an exact derivative, albeit with additive noise. However, stimulus modulation distorts (broadens) the observed shape of the underlying signal. This modulation broadening can be accounted for as part of the convolution with the (usually) Gaussian kernel describing the linewidth of the underlying spectral features. This is sufficient at low modulation depths, but does not correctly describe the shape of the signal when the modulation broadening is the dominant in determining the shape of the signal. The detailed effect of stimulus modulation on the resulting signal will be explored in the next section.

\section{THEORY}

In this section we will derive a convolutional representation of the effects of the modulation on the measured signal, ultimately arriving at Equations 36 and 37. The derivation is presented in great detail, with the intention of highlighting all of the techniques and assumptions involved, and in such a way as to allow its adaptation for other settings. All the
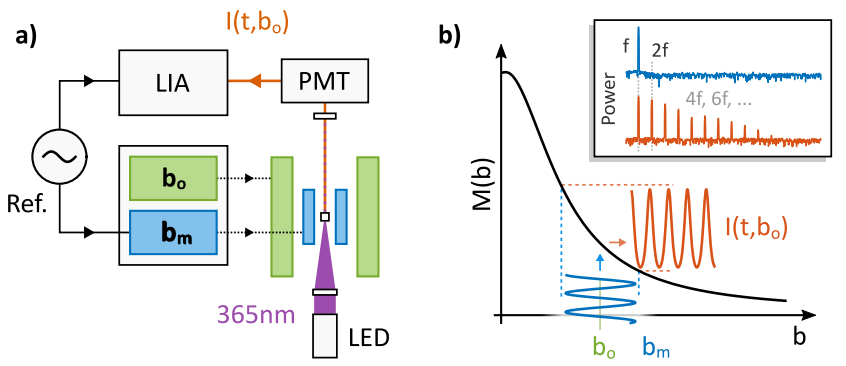

Figure 1. a) Schematic of the ModMARY experimental apparatus. The sample is excited by a collimated LED and its fluorescence is measured by a photomultiplier tube (PMT) after passing through an excitation filter. The sample is contained within 2 parallel sets of magnetic field coils which produce the offset $b_{o}$ and the modulated $b_{m}$ magnetic field components. The LIA and the $b_{m}$ power supply share a common frequency reference. b) Representation of the MARY curve acting as a transfer function $M$ from magnetic field (abscissa) to fluorescence intensity (ordinate). The magnitude of the modulated fluorescence signal $I$ shown is approximately proportional to the derivative of $M$ in the modulation region. However, $I$ can undergo harmonic distortion due to the nonlinearity of $M$. This comparison is easier to see in the power spectral density spectra of the (artificial) input field (top) and $I$ (bottom) were one can see overtones of the fundamental frequency $f$.

symbols used are defined throughout the text, but a glossary is also provided at the end of the main text. The reader is encouraged to pay particular attention to Sec. II C which explores the relation between a series expansion of a transfer function and the harmonic distortion it causes. Although presented in the context of ModMARY spectroscopy, this is the core idea behind any kind of overtone spectroscopy technique.

\section{A. Lock-in detection}

In order to understand the effect of modulation on the signal, we need a model of the detector. The lock-in amplifier (LIA) measures the amplitude of an oscillation component of the input voltage $I$, which is both frequency and phase coherent with the reference oscillation $R$, in this case the reference voltage for the modulation coils power supply. This is achieved by direct multiplication of $I$ and $R$ in the time domain (mixing). This corresponds to convolution in the frequency domain and is also referred to as demodulation. The zero frequency (DC) component of the mixer output is the root-mean-square amplitude of $I$. It can be recovered with the use of a low-pass (LP) filter ${ }^{24}$ to give the output voltage $S$. A characteristic setting of a LIA is its time constant $\tau$, effectively its averaging time. The quantity $1 / \tau$ is the width of the corresponding LP filter applied to the demodulated signal. The action of a simple resistor-capacitor 
(RC) LP filter can be represented by the integral: ${ }^{25}$

$$
y=\frac{1}{\tau} \int_{-\infty}^{0} e^{t / \tau} x(t) \mathrm{d} t
$$

where $y$ is the filtered version of input signal $x(t)$ with LP filter of width $1 / \tau$. In order to account for a possible phase difference between $I$ and $R$, most LIAs implement a quadrature detection scheme whereby the input signal is split into two identical paths. One path mixes $I$ with $R$, and the other with $R$ delayed by quarter of an oscillation of $f_{m}$. The inphase signal can be reconstructed from the resulting measurement (see Sec. III B). $R$ can therefore be represented as a complex exponential where the real and imaginary components correspond to the quadrature channels of the LIA:

$$
R(t)=\cos (h \omega t)+i \sin (h \omega t)=\exp \left(i \frac{h \eta}{\tau} t\right)
$$

It will prove convenient to work with the "wavenumber" $\eta$, a proxy for the number of radians of oscillation of $R$ in a time constant $\tau$ defined as $\tau \omega=\eta$. We have also introduced an integer harmonic number $h$ which, for the time being, should be considered to be equal to 1 and will be useful later. The output of a LIA can be modelled by the following integral:

$$
S_{h}\left(b_{o}\right)=\int_{-\infty}^{0} D_{h}(t) I\left(b_{o}, t\right) \mathrm{d} t
$$

where $D_{h}$ is defined as:

$$
D_{h}(t)=\frac{\sqrt{2}}{\tau} \exp \left(\frac{1+i h \eta}{\tau} t\right)
$$

Both of the quadrature components of the reference waveform, the normalisation constant, and the exponential detection window of the LP filter have been combined in a single exponential term - the detection kernel $D_{h}$. The factor of $\sqrt{2}$ accounts for the fact that the output of the LIA is proportional to the root-mean-square amplitude of the input signal. ${ }^{13}$

The case of $\eta \gg 1$ and $h>0$ corresponds to the period of the reference oscillation $(R)$ being much shorter than the LP filter averaging time $\tau$. Then, $D_{h}$ by itself integrates to 0 :

$$
\int_{-\infty}^{0} D_{h} \mathrm{~d} t= \begin{cases}\sqrt{2} & h=1 \\ 0 & \text { otherwise }\end{cases}
$$

$h=0$ can be thought of as the special case of direct signal detection with no modulation (DC). $D$ is represented pictorially in Fig. 3.

\section{B. Single tone input}

It will prove useful to understand how the LIA output, defined in Eq. 3, behaves as a function of a single tone, $1 \mathrm{~V}$ root-mean-square (rms), input:

$$
I(t)=\sqrt{2} \cos \left(\omega_{I} t+\theta\right)=\sqrt{2} \cos \left(\eta_{I} t / \tau+\theta\right)
$$

where $\omega_{I}$ and $\eta_{I}$ are the (angular) frequency, and wavenumber of the input tone. For $\eta \gg 1$, Eq. 3 integrates to:

$$
S=e^{-i \theta} \frac{1-i\left(\eta-\eta_{I}\right)}{1+\left(\eta-\eta_{I}\right)^{2}}
$$

which is a Lorentzian lineshape with height 1 , centered at $\eta_{I}$. The phase $(\theta)$ of the input rotates between the real and imaginary components of the Lorentzian. The real part corresponds to the the absorptive and the imaginary to the dispersive components of the Lorentzian. ${ }^{26}$ The above equation can be simplified if we take the square modulus to obtain the power, as opposed to the magnitude, of the signal. The phase dependence of the quadrature channels vanishes, and Eq. 7 simplifies to:

$$
|S|^{2}=\frac{1}{1+\left(\eta-\eta_{I}\right)^{2}}
$$

which is the response of the LIA to a unit rms amplitude, high frequency $(\eta \gg 1)$ tone with wavenumber $\eta_{I}$ as a function of the wavenumber of the reference waveform $\eta$. This is a notch with the magnitude of the rms of the input centered at $\eta_{I}$. The width of $|S|^{2}$ at half-magnitude $(-3 \mathrm{~dB})$ is one wavenumber. This corresponds to the width of an RC filter with width $1 / \tau$.

\section{Transfer function}

Having described the action of the LIA, we shall now consider the ModMARY experiment. The total magnetic field experienced by the sample is the sum of the static and oscillating magnetic fields:

$$
b(t)=b_{o}+b_{m} \cos (\omega t+\theta)
$$

The oscillating input of the LIA in the ModMARY experiment arises due to the MARY curve acting akin to a transfer function $M$ from field to fluorescence intensity (Fig. 1). In Sec. III B (data analysis), $M$ will modelled by a (scaled and shifted) Lorentzian, or double Lorentzian lineshape (see Eq. 39). The derivation presented in here will, however, make only the most basic assumptions about $M$ - that it is a smooth, continuous function, well-defined over the entire domain of interest. Furthermore, the shape of $M$ will be assumed to be time invariant. This last assumption could be relaxed in more detailed analysis.

If $M$ is the exact shape of the MARY curve, then the LIA input can be expressed as:

$$
I(b)=I_{0}+M(b)=I_{0}+M\left(b_{o}+b_{m} \cos (\omega t+\theta)\right)
$$

where $I_{0}$ is the baseline fluorescence component which has not been magnetically altered. The MARY curve, $M$, can be approximated locally by using a Taylor expansion of $M(b)$ 
about point $b_{o}$. This can be done for sufficiently small $b_{m}$, such that $b \approx b_{o}$ (see Eq. 9). Substituting this Taylor expansion of $M$, up to the $1^{\text {st }}$ order term, into Eq. 10, gives:

$$
\begin{aligned}
I\left(b_{o}, t\right) & =I_{0}+M\left(b_{o}\right)+M^{\prime}\left(b_{o}\right)\left(b-b_{o}\right)+\mathcal{O}\left(M^{n \prime}\left(b_{o}\right)\right)_{n>1} \\
& \approx\left(I_{0}+M\left(b_{o}\right)\right)+M^{\prime}\left(b_{o}\right) b_{m} \cos (\omega t+\theta)
\end{aligned}
$$

where' indicates a derivative with respect to magnitude of the magnetic field applied $b$, and $\mathcal{O}$ is the order function signifying higher order terms. This signal consists of the DC component $I_{0}+M\left(b_{o}\right)$ and the AC component which, in this case, is a single tone at $\omega$. This is an approximation of the input into the LIA. The corresponding output can be found by applying the LP filter equation. Substituting Eq. 11 into Eq. 3, and setting $h=1$ gives:

$$
\begin{aligned}
S\left(b_{o}\right) & =\left(I_{0}+M\left(b_{o}\right)\right) \int_{-\infty}^{0} D_{1} \mathrm{~d} t+M^{\prime}\left(b_{o}\right) b_{m} \int_{-\infty}^{0} D_{1} \cos (\omega t+\theta) \mathrm{d} t \\
& =e^{-i \theta} \frac{\sqrt{2}}{2} b_{m} M^{\prime}\left(b_{o}\right)
\end{aligned}
$$

The DC component of $I$ has been filtered out, and the detected signal is therefore the magnitude of the input signal - the derivative of the MARY curve $M^{\prime}$ scaled by the modulation amplitude $\sqrt{2} b_{m} / 2$ and phased between the detection channels by angle $\theta$.

The higher the magnitude of $b_{m}$, the higher the signal, but also the more we depart from the regime where we can truncate higher order terms of the expansion in Eq. 10. This approximation fails even for small $b_{m}$ at, and close to, turning points where $M^{\prime}$ is by definition small, but $M^{\prime \prime}$ is large. This can be seen by plotting the accuracy of the local Taylor approximation of a Lorentzian lineshape with truncations at progressively higher terms. The log of local error of the expansion up to the $n^{\text {th }}$ term $\left(\operatorname{LLE}_{n}\right)$ has been plotted in Fig. 2. It can be seen that when $L_{L} E_{1}$ is highest, $L_{L E}$ is lowest. The successive even terms of the Taylor expansion contribute the most where the preceding odd one contributes the least, and vice versa. We can mitigate the problem of inaccurate representation of $I$ by leaving one more term in the expansion, giving:

$$
\begin{aligned}
I\left(b_{o}, t\right) & =I_{0}+M\left(b_{o}\right)+M^{\prime}\left(b_{o}\right)\left(b-b_{o}\right)+\frac{1}{2} M^{\prime \prime}\left(b_{o}\right)\left(b-b_{o}\right)^{2}+\mathcal{O}_{n>2}\left(M^{n}\left(b_{o}\right)\right) \\
& \approx\left(I_{0}+M\left(b_{o}\right)+\frac{M^{\prime \prime}\left(b_{o}\right)}{4} b_{m}^{2}\right)+M^{\prime}\left(b_{o}\right) b_{m} \cos (\omega t+\theta)+\frac{M^{\prime \prime}\left(b_{o}\right)}{4} b_{m}^{2} \cos (2(\omega t+\theta))
\end{aligned}
$$

There are now two tones, one at $\omega$ with phase $\theta$, and one at $2 \omega$ with phase $2 \theta$. The main component of an $n^{\text {th }}$ harmonic of $\omega$ is the $n^{\text {th }}$ derivative of $M$. The magnitude of the $n^{\text {th }}$ harmonic also scales as $\mathcal{O}\left(b_{m}^{n}\right)$, although this does not necessarily translate into signal magnitude. This is because the magnitude of higher derivatives of physically useful $M$ 's tend to vanish as $n$ increases, since such $M$ 's tend to be bounded and limited to a certain region (e.g. electron paramagnetic resonance spectra, spectral extinction coefficients, emission profiles). Note also that $M^{\prime \prime}$ contributes to the DC component but will, as previously, be discarded by LP filtering.

Substituting Eq. 13 into Eq. 3 gives:

$$
\begin{aligned}
S_{h}\left(b_{o}\right) & =M^{\prime}\left(b_{o}\right) b_{m} \int_{-\infty}^{0} D_{h} \cos (\omega t+\theta) \mathrm{d} t \\
& +\frac{M^{\prime \prime}\left(b_{o}\right)}{4} b_{m}^{2} \int_{-\infty}^{0} D_{h} \cos (2 \omega t+2 \theta) \mathrm{d} t
\end{aligned}
$$

We can now use the previously introduced harmonic number $h$ to tune into either of the two tones, since first or second integral can be set to 0 by setting $h=2$ or $h=1$ respectively. The first and second harmonic signals are therefore given by:

$$
\begin{gathered}
S_{1}\left(b_{o}\right)=e^{-i \theta} \frac{\sqrt{2}}{2} b_{m} M^{\prime}\left(b_{o}\right) \\
S_{2}\left(b_{o}\right)=e^{-i 2 \theta} \frac{\sqrt{2}}{8} b_{m}^{2} M^{\prime \prime}\left(b_{o}\right)
\end{gathered}
$$

As discussed above, the first harmonic signal is proportional to the first derivative. The second harmonic signal (or first overtone) is proportional to the second derivative. It can therefore be used to reconstruct the MARY curve in regions where $M^{\prime}$ is small.

In principle, the greater the value of $b_{m}$, the greater the nonlinearity of $M$ in the region swept by the modulation $\left(b_{o}-\right.$ $b_{m}$ to $\left.b_{o}+b_{m}\right)$. This means that higher order expansions are needed to approximate $M$ correctly, and therefore higher overtone signals are detectable from the experiment.

As all the harmonics scale with powers of $b_{m}$, they become larger and easier to detect as $b_{m}$ increases. However, it becomes much more problematic to interpret the data for high $b_{m}$. Just as the $2^{\text {nd }}$ order expansion had an effect on the 0 th order term (DC), the $3^{\text {rd }}$ order expansion will have an effect on the $1^{\text {st }}$ order term $S_{1}$. In general, each $n^{\text {th }}$ order term contributes to the harmonics $n, n-2, n-4, \ldots$ etc. ${ }^{27}$ This results in broadening of the corresponding signals as $b_{m}$ sweeps over larger and larger regions (see Fig. 4). 


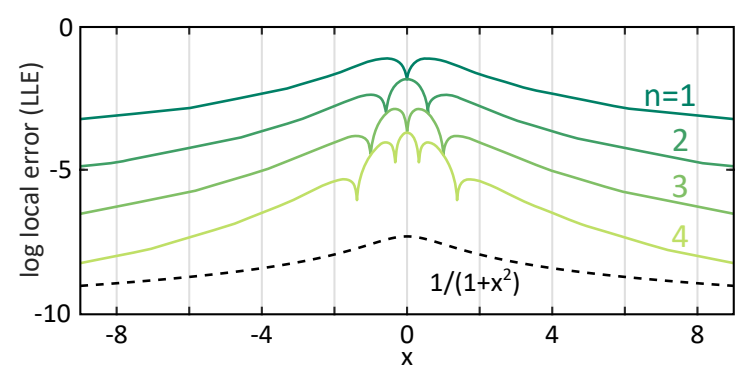

Figure 2. Log local error (LLE) in the successive Taylor expansions of a Lorentzian lineshape $L(x)=1 /\left(1-x^{2}\right)$ (dashed black line). In this context, $\operatorname{LLE}_{n}$ is defined as $\log \left(\max \left(\left|L-L_{n}\right|\right)\right)$, where $L_{n}$ is the Taylor expansion of $L$ up to and including the $n^{\text {th }}$ derivative term in the region $x \pm 0.1$ (see supplementary information for details). $\mathrm{LLE}_{n}$ can be treated as inversely proportional to the information about the original function $L$, carried by the $n^{\text {th }}$ term (and, by extension, the $n^{\text {th }}$ harmonic) of the expansion. The region where the first local Taylor approximation of $L$ commits the largest error (peak in $L_{1}$ at $|x| \approx 1$ ) is the region of high curvature of $L$. This region is, in turn, best approximated by the second local Taylor expansion (dip in $\mathrm{LLE}_{2}$ at $|x| \approx 1$ ). It shows how the second harmonic detection helps with reconstruction of the high curvature (i.e. high $2^{\text {nd }}$ derivative) regions of $M$.

\section{Periodic split}

To proceed with the derivation, we need to split the integral in Eq. 3 into a sum of integrals over the units of a full period of $\omega$, the fundamental reference frequency (see Fig. 3). Furthermore, we change the variable of integration from $t$ to $\mathfrak{t}=\omega t / 2 \pi$, effectively time in units of one full period of $\omega$. The split integral can be written as:

$$
\begin{array}{r}
S_{h}=\int_{-\infty}^{0} D_{h} I \mathrm{~d} t=\sum_{j=0}^{-\infty} \int_{\frac{(j-1) \pi}{\omega}}^{\frac{j \pi}{\omega}} D_{h} I \mathrm{~d} t \\
=\frac{2 \sqrt{2} \pi}{\eta} \sum_{j=0}^{-\infty} \int_{j-1}^{j} \exp \left(\frac{2 \pi}{\eta} \mathfrak{t}\right) \exp (2 \pi i h \mathfrak{t}) I \mathrm{~d} \mathfrak{t}
\end{array}
$$

where the explicit dependence of $S_{h}$ on $b_{o}$ has been dropped to simplify notation.

For each individual period, the exponential envelope term $\exp \left(\frac{2 \pi}{\eta} \mathfrak{t}\right)$ can be expressed as a product of the average height of the envelope, its shape:

$\exp \left(\frac{2 \pi}{\eta} \mathfrak{t}\right) \stackrel{j^{\text {th }} \text { period }}{\longrightarrow} \exp \left(\frac{2 \pi}{\eta}(j-1 / 2)\right) \times \exp \left(\frac{2 \pi}{\eta}(\mathfrak{t}+1 / 2)\right)$

The first term is the height of $\exp \left(\frac{2 \pi}{\eta} \mathfrak{t}\right)$ half way through $j^{\text {th }}$ period:

$$
J(j, \eta)=\exp \left(\frac{2 \pi}{\eta}(j-1 / 2)\right)
$$

and the second is the exponential envelope shape:

$$
E(\mathfrak{t}, \eta)=\exp \left(\frac{2 \pi}{\eta}(\mathfrak{t}+1 / 2)\right) \stackrel{\eta \gg 1}{=} 1
$$

$J$ is independent of $t$, and so can be taken out of the integral as a constant. The entire integrand is now independent of $j$ and so the entire integral, in turn, can be taken out of the summation. Inserting the definitions from Eq. 18 and 19 into Eq. 16, and rearranging as described, yields:

$$
S_{h}=\frac{2 \sqrt{2} \pi}{\eta} \sum_{j=0}^{-\infty}\{J(j, \eta)\} \int_{-1}^{0} E(\mathfrak{t}, \eta) \exp (2 \pi i h \mathfrak{t}) I \mathrm{~d} \mathfrak{t}
$$

The limits of the integral have been changed accordingly, as the period-wise shift back in time $(j)$ is fully accounted for by $J$. This can be done since, be definition, the integrand is periodic in $t$ with a period of 1 . The sum over $J$ is bound and evaluates to a hyperbolic cosecant:

$$
\sum_{j=0}^{-\infty} J(j, \eta)=\sum_{j=0}^{-\infty} \exp \left(\frac{2 \pi}{\eta}(j-1 / 2)\right)=\frac{1}{2} \operatorname{csch}\left(\frac{\pi}{\eta}\right)
$$

Eq. 20, therefore, becomes:

$$
S_{h}=k(\eta) \int_{-1}^{0} E(\mathfrak{t}, \eta) \exp (2 \pi i h \mathfrak{t}) I \mathrm{~d} \mathfrak{t}
$$

where $k$ is the scaling constant:

$$
k(\eta)=\frac{\sqrt{2} \pi}{\eta} \operatorname{csch}\left(\frac{\pi}{\eta}\right) \stackrel{\eta \gg 1}{=} \sqrt{2}
$$

\section{E. Change of domain}

We have simplified an infinite integral to one over a single period of the fundamental frequency, and we know how the applied magnetic field changes in time. We can therefore use this knowledge to change the variable of integration from time to magnetic field - the natural domain of the signal measured. To do this we to start by defining a modulation parameter $\mathfrak{m}$ as:

$$
\mathfrak{m}=\cos (2 \pi \mathfrak{t}+\theta)
$$

where $\mathfrak{m}$ is the oscillating part of the magnetic field scaled to unit amplitude, and $\omega t \rightarrow 2 \pi t$. The corresponding equations to change the variable of integration are:

$$
\begin{aligned}
& \mathfrak{t}=\frac{1}{2 \pi} \cos ^{-1}(\mathfrak{m})-\frac{\theta}{2 \pi} \\
& \mathrm{d} \mathfrak{t}=\mp \frac{1}{2 \pi \sqrt{1-\mathfrak{m}^{2}}} \mathrm{dm}
\end{aligned}
$$



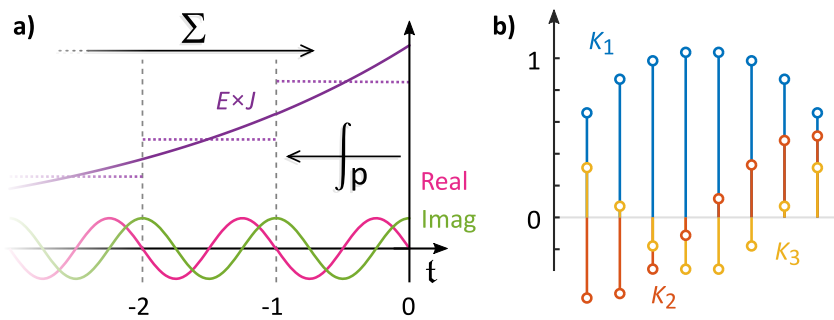

Figure 3. a) Pictorial representation of the components of the detection kernel ( $D$, Eq. 4), shown as a function of relative time $t$. The purple line represents the integrator window component of the detection kernel ( $D$ ) - exponential with time constant $\tau$. The dashed purple line corresponds to the average height of this window in each period of the modulation ( $J$, Eq. 18). The solid purple line is obtained by multiplying $J$ with the exponential envelope ( $E$, Eq. 19) within each period. Real (green) and imaginary (pink) quadrature components of the reference waveform are also shown. The arrows demonstrate the splitting of the integral from Eq. 16 into an infinite sum, $\Sigma$, of integrals over full periods of the reference, $\int_{\mathrm{p}}$. b) The first three modulation kernels $K_{h}$, sampled with 10 points and scaled to conserve signal magnitude under convolution. Only the 8 middle samples are shown, since the edge samples evaluate to $0 . K_{2}$ is acting akin to the differentiation kernel used in finite-difference numerical differentiation, combined with moving-average smoothing. Correspondingly, $K_{3}$ is the second derivative. Note that these kernels operate on the derivative of $M$.

where the sign of the derivative changes between the left and right half-periods of $\omega$. Changing the variable from $t$ to $\mathfrak{m}$ in Eq. 22 results in:

$$
S_{h}\left(b_{o}\right)=\frac{k}{2 \pi} e^{-i h \theta} \int_{\text {period }} \mp E \frac{\exp \left(i h \cos ^{-1}(\mathfrak{m})\right)}{\sqrt{1-\mathfrak{m}^{2}}} M \mathrm{dm}
$$

where the integration range has not been explicitly specified yet as it needs further careful attention. $I(t)$ has been replaced by $M(b)=M\left(b_{o}+b_{m} \mathfrak{m}\right)$, the shape of the MARY curve in the range $\left[b_{o}-b_{m}, b_{o}+b_{m}\right]$. Since only the integrand is complex, the integration in Eq. 26 can be carried out separately for the real and the imaginary components.

The real part of the integral from Eq. 26 is:

$$
\mathbb{I}_{\text {Re }}=\int_{\text {period }} \mp E \frac{\cos \left(h \cos ^{-1}(\mathfrak{m})\right)}{\sqrt{1-\mathfrak{m}^{2}}} M \mathrm{dm}
$$

The limits of the integral are problematic since the inverse function of $\cos (x)$ is multivariate. The integral above must therefore be evaluated piecewise. Doing this for arbitrary $\eta$ and $h$ for both real and imaginary parts would give $2(2 h+1)$ integrals. Proceeding with this would allow the derivation of a general solution, even far from the limit of $\eta \gg 1$. This analysis is complicated and unnecessary for this work. From now on we shall assume $\eta \gg 1$. In this limit we can use the previously established approximations $E \approx 1$ over the entire period $\omega$, and $k \approx \sqrt{2}$. Applying these limits to Eq. 27 yields:

$$
\mathbb{I}_{\mathrm{Re}}=\int_{1}^{-1} \frac{-\mathcal{T}_{h}(\mathfrak{m})}{\sqrt{1-\mathfrak{m}^{2}}} M \mathrm{~d} \mathfrak{m}+\int_{-1}^{1} \frac{\mathcal{T}_{h}(\mathfrak{m})}{\sqrt{1-\mathfrak{m}^{2}}} M \mathrm{~d} \mathfrak{m}
$$

where $\mathcal{T}_{h}(\mathfrak{m})$ has been recognised as a Chebyshev polynomial of the first kind, of order $h$, over $\mathfrak{m}$ :

$$
\mathcal{T}_{h}(\mathfrak{m})=\cos \left(h \cos ^{-1}(\mathfrak{m})\right)
$$

For even $h$, the limits of the integral do not matter as the integrand is symmetric about 0 . For odd $h$ the limits of the right integral can be swapped by changing the sign and so in each case the two integrals are equal. The real part of the integral from Eq. 26 can therefore be written as:

$$
\mathbb{I}_{\mathrm{Re}}=2 \int_{-1}^{1} \frac{\mathcal{T}_{h}(\mathfrak{m})}{\sqrt{1-\mathfrak{m}^{2}}} M \mathrm{dm}
$$

A similar argument can be applied to the imaginary part of the integral. Here, the corresponding term is a Chebyshev polynomial of the second kind of order $h-1$, over $\mathfrak{m}$, and can be written as:

$$
\pm \mathcal{U}_{h-1}(\mathfrak{m}) \sqrt{1-\mathfrak{m}^{2}}=\sin \left(h \cos ^{-1}(\mathfrak{m})\right)
$$

The sign depends on the domain of the $\cos ^{-1}(\mathfrak{m})$ term. It is positive for $\cos ^{-1}(\mathfrak{m}) \in[-\pi, 0]$ and negative for $\cos ^{-1}(\mathfrak{m}) \in$ $[-2 \pi,-\pi]$. This, conveniently, corresponds to the way we split the integral, and the additional sign change means the two halves cancel each other out. The imaginary part of the integral from Eq. 26 is:

$$
\mathbb{I}_{\text {Im }}=\int_{1}^{-1}-\mathcal{U}_{h-1}(\mathfrak{m}) M \mathrm{~d} \mathfrak{m}-\int_{-1}^{1} \mathcal{U}_{h-1}(\mathfrak{m}) M \mathrm{~d} \mathfrak{m}=0
$$

Combining both real (Eq. 30) and imaginary (Eq. 32) parts of the integral, Eq. 26 becomes:

$$
S_{h}\left(b_{o}\right)=\frac{\sqrt{2}}{\pi} e^{-i h \theta} \int_{-1}^{1} \frac{\mathcal{T}_{h}(\mathfrak{m})}{\sqrt{1-\mathfrak{m}^{2}}} M\left(b_{o}+b_{m} \mathfrak{m}\right) \mathrm{d} \mathfrak{m}
$$

This integral in the field domain can be interpreted as a convolution of scaled $M$ with a Chebyshev kernel. $M$ is required to be scaled "horizontally" in the field direction such that $b_{m}=1$. This can always be done without loss of generality.

This form of the expression for the LIA signal can already be used to obtain the analytical result for the shape of the resulting signal. The only remaining problem (at least for numerical analysis) is that the term $\mathcal{T}_{h}(\mathfrak{m})\left(1-\mathfrak{m}^{2}\right)^{-1}$ has an infinite amplitude as $|\mathfrak{m}| \rightarrow 1$. This can be resolved by simplifying the integral from Eq. 33 using integration by parts. 
Integrating the Chebyshev term and differentiating $M$ with respect to $b$ gives:

$$
\begin{aligned}
S_{h}\left(b_{o}\right)=\frac{\sqrt{2}}{\pi h}\{ & M \int_{-1}^{1} \mathcal{U}_{h-1}(\mathfrak{m}) \sqrt{1-\mathfrak{m}^{2}} \mathrm{dm} \\
& \left.+\int_{-1}^{1} \mathcal{U}_{h-1}(\mathfrak{m}) \sqrt{1-\mathfrak{m}^{2}} M^{\prime} \mathrm{d} \mathfrak{m}\right\}
\end{aligned}
$$

As the first integral is 0 for $h \geq 1$, the above equation can be simplified to:

$$
S_{h}\left(b_{o}\right)=\int_{-1}^{1} K_{h}(\mathfrak{m}) M^{\prime}\left(b_{o}+b_{m} \mathfrak{m}\right) \mathrm{d} \mathfrak{m}
$$

or, more compactly:

$$
S_{h}\left(b_{o}\right)=K_{h} \otimes M^{\prime}
$$

where $\otimes$ represents the operation of convolution and $K_{h}$ is the convolution kernel:

$$
K_{h}= \begin{cases}\exp (-i h \theta) \frac{\sqrt{2}}{\pi h} \mathcal{U}_{h-1}(\mathfrak{m}) \sqrt{1-\mathfrak{m}^{2}} & \mathfrak{m} \in[-1,1] \\ 0 & \text { otherwise }\end{cases}
$$

It can be seen that the phase of the reference only rotates the signal between the real and imaginary planes. When considering a well phased signal $(\theta=0)$, the exponential term is equal to 1 and $K_{h}$ and $S_{h}$ are purely real.

$M^{\prime}$ is the field derivative of the transfer function which describes the magnetic field sensitivity of the sample of interest, and $K_{h}$ is an analytical modulation kernel (Eq. 37) which encapsulates both the modulation broadening and any further derivative action (for higher signal overtones). Representative 10-point convolution kernels for $h$ from 1 to 3 have been plotted in Fig. 3. The amplitude of the modulation is used to scale the field axis of $M^{\prime}$ such that $b_{m}=1$. Equivalently, $M^{\prime}$ could be left unchanged and $K_{h}$ scaled to go from $-b_{m}$ to $+b_{m}$. Note that, as long as $\eta \gg 1, K_{h}$ does not depend on modulation frequency $f_{m}$ nor on the LIA time constant $\tau$.

This formulation of the ModMARY signal, and indeed any derivative spectroscopy signal, is immensely useful. Not only does this give us an exact analytical shape of the obtained derivative signal (including the distortion due to modulation), but even when that analytical result is hard/impossible to calculate, numerical convolution is fast, even for a very large number of data points. This allows fitting of any $M$ to the derivative signal as long as the modulation amplitude is known. Moreover, this formulation allows us to interpret overtone signals easily. As discussed in Sec. II C, higher overtone signals often contain valuable information about regions of interest of $M$ which, in the case of this work, is the LFE region of the MARY curve.
The utility of this method will be demonstrated in the following section with an experimental study and fitting of a well known magnetosensitive model system (Py/DCB) at different modulation amplitudes.

\section{EXPERIMENT AND METHODS}

\section{A. Experimental apparatus}

A schematic of the experiment can be seen in Fig. 1. The sample is excited by a $365 \mathrm{~nm}$ LED (Thorlabs M365L2) which is collimated into a $\sim 0.5 \mathrm{~cm}$ wide beam and directed onto the $1 \mathrm{~cm}$ quartz cuvette (Hellma Analytics, 130-10-40, $10 \times 10 \mathrm{~mm}$ ) through a reflective prism. The sample was flowed through the sample cell at $10 \mu \mathrm{Lmin}^{-1}$ and allowed to reach a steady state before the measurement. The effective radiant power at the sample has been estimated as $\sim 100 \mathrm{~mW}$. The fluorescence is collected with a lightguide and directed to the photocathode of a photomultiplier tube (PMT, Hamamatsu) through a $455 \mathrm{~nm}$ LP filter (Comar optics, 455 GY 25). The PMT has been set in a $\mu$-metal box and placed far from the magnetic field coils to shield it from the influence of the magnetic fields. Two sets of magnetic field coils are placed around the sample, one for the offset and one for the modulated components of the applied magnetic field. The fields applied by the coils are parallel to one another and each set has an independent power supply (Physical and Theoretical Chemistry Laboratory (PTCL), University of Oxford, Electronics workshop).

A hall probe gaussmeter (PTCL electronics) is fixed in the sample cell holder to monitor the field applied over the course of the experiment. The probe has been calibrated against a commercial Gaussmeter (Lakeshore 425 with HST4 probe), with the probe placed in the sample cell holder, which also accounts for any field distortion due to the cell housing.

The PMT outputs up to $15 \mathrm{~V}$ DC at maximum light exposure. The output was kept at $\sim 10 \mathrm{~V}$ by adjusting the gain before starting the experiment. The gain was then kept constant. The signal from the PMT is split into separate paths by two unity-gain voltage buffers (PTCL electronics). One path is AC coupled and demodulated by two analog LIAs (Stanford Research Systems 510), corresponding to two quadrature channels. Both LIAs have been calibrated in the same way and, except for the $\pi / 2$ phase shift, are identical to one another. The other path goes through a calibrated voltage divider to bring the output of the PMT into the $10 \mathrm{~V}$ range.

The (scaled) PMT DC voltage, the analog output of the LIAs, and the gaussmeter signal are digitised by a data acquisition card (DAQ, National Instruments, PCIe-6323) at $2 \mathrm{kHz}$ sample rate in $50 \mathrm{~ms}$ consecutive snapshots, giving a data acquisition rate of $20 \mathrm{~Hz}$. The PMT voltage was recorded through a voltage divider to ensure that the DAQ card input was not overloaded. Each snapshot of the gaussmeter signal was further processed by converting the measured 
voltage to calibrated magnitude of the magnetic field using $\mathrm{a} 4^{\text {th }}$ order polynomial, and measuring the detected $f_{m}$ and $b_{m}$ with the FFT based single tone measurement search algorithm. ${ }^{28}$ The DAQ card was also used to output an analog $\pm 10 \mathrm{~V}$ signal controlling the offset field power supply. The negative control voltage corresponding to the opposite current flow and hence the magnetic field being applied in the opposite direction.

The experiment was controlled by a custom written program (National Instruments, LabVIEW, 2015b). The magnetic field was constantly adjusted using a fast ZieglerNicolas PID controller to ensure any effects of magnet hysteresis were negligible. ${ }^{29}$ Moreover, the field was swept in an up-down-up fashion between \pm maximum field values to ensure symmetrical signal with no artifacts. At each field point, the experiment paused for at least 3 times the time constant of the LIAs before acquiring the $50 \mathrm{~ms}$ data sample. Each data point consists of the mean and standard deviation of each of the sample of the LIAs outputs. The PMT voltage, the gaussmeter reading, measured $f_{m}$ and measured $b_{m}$ were also recorded.

The offset magnetic field applied to the sample was in the range of $\pm 40 \mathrm{G}$, with an accuracy of better than $0.1 \mathrm{G}$. The average field settling time following change of field set point in a sweep was $400 \mathrm{~ms}$ (see supplementary information). Both the $1^{\text {st }}$ and the $2^{\text {nd }}$ harmonic ( $h_{1}$ and $h_{2}$ respectively) signals have been measured for each dataset shown. The modulation amplitude was always kept constant throughout the course of the experiment. The achievable range of amplitudes was dependent on $f_{m}$, due to the coils' impedance increasing at higher frequency. For all of the following experiments the modulation frequency was kept at $193 \mathrm{~Hz}$, except for $20 \mathrm{G}$ modulation depth where is was set to $71 \mathrm{~Hz}$ (both prime). These frequencies were used such that neither the modulated fluorescence signal, nor its overtones overlap significantly with electrical interference from power lines $(50 \mathrm{~Hz}$ fundamental). In practise this interference was found to be significant only at modulation frequencies close to 50 and $100 \mathrm{~Hz}$. Higher modulation frequencies were not employed since no impedance matching circuit was used, and so high frequency would limit the achievable modulation depth range. Note that, by convention, ModMARY spectroscopy refers to the "modulation depth" which is twice the modulation amplitude and corresponds directly to the region swept by the oscillating component of the magnetic field.

The reference frequency for the LIAs and the power supply of the modulation coils was provided by an external waveform generator (Rigol DG1022). The sample cell holder block was temperature controlled by a recirculating chiller ( $240 \mathrm{~W}$, Grant Instruments, LT ecocool 100). The steady state temperature at the return of the chiller was just above room temperature $\left(23^{\circ} \mathrm{C}\right)$. The equipment has been checked extensively to ensure that the magnetic field does not produce artifacts in the data (see supplementary information).

\section{B. Analysis}

The data acquired from each of the quadrature channels of the LIA $S_{x}$ and $S_{y}$, has been phased by minimizing the magnitude of the imaginary component with respect to the complex angle or rotation $\theta$. The result is a phased signal $S_{p}$ which preserves the sign of the original data:

$$
\begin{gathered}
\bar{\theta} \leftarrow \min _{\theta} \sum \operatorname{Im}\left[\left(S_{x}+i S_{y}\right) \times \exp (i \theta)\right]^{2} \\
S_{p}=\left(S_{x}+i S_{y}\right) \times \exp (i \bar{\theta})
\end{gathered}
$$

In order to reconstruct the underlying MARY curve (the fluorescence magnetosensitivity transfer function $M$ ) we can fit the $h_{1}$ and $h_{2}$ data to Eq. 36 . We can do this by creating a parametrized model of $M$, calculating the appropriate convolution kernel $\left(K_{h}\right)$ from the experimental parameters, and performing a least-squares fit.

We use a sum of two Lorentzian lineshapes of opposite signs to model $M$. The model $M_{L}$ is given by:

$$
M_{L}(x \mid \boldsymbol{p})=4 x^{2}\left(\frac{p_{2}}{p_{4}^{2}+4 x^{2}}-\frac{p_{1}}{p_{3}^{2}+4 x^{2}}\right)
$$

where $\boldsymbol{p}$ is the vector of fit parameters, the two amplitudes are given by $p_{1}$ and $p_{2}$, and the two widths of the high and low field components of $M_{L}$ by are given by $p_{3}$ and $p_{4}$ respectively.

An analytical gradient for $M_{L}$ is easy to calculate, but in the interest of generality, and to benchmark performance for an arbitrary $M$, a numerical gradient of densely sampled $M_{L}$ was used instead. $M_{L}^{\prime}$ was convolved with the appropriately sampled and scaled $K_{h}$. The additional scaling was necessary to account for the discrete sampling of $K_{h}$ (see supplementary information). Special care was taken to sample $M$ over a wide enough region for the convolution with $K_{h}$ to be valid. This densely sampled result was then interpolated onto the data axis, and the mean square error between the fit and the data was evaluated and optimised as a function of $\boldsymbol{p}$. For fitting multiple datasets simultaneously, in order to account for varied signal magnitude between different modulation amplitudes and harmonics, the residuals for each dataset were scaled by their root-mean-square magnitude. The fitted vector of model $\left(M_{L}\right)$ parameters $\overline{\boldsymbol{p}}$ was obtained by:

$$
\overline{\boldsymbol{p}} \leftarrow \min _{\boldsymbol{p}}\left[\sum\left(S_{p}-K_{h} \otimes M_{L}^{\prime}(\boldsymbol{p})\right)^{2} \div \sum S_{p}^{2}\right]
$$

The optimisation was performed with a Nelder-Mead search algorithm. ${ }^{30}$ It is worth noting that an improper implementation of the convolution can lead to artefacts appearing in the spectrum due to zero-padding of the signal beyond its domain. In the worst case scenario, these artifacts can appear like features of interest, for example the LFE feature. In order to prevent this, $M$ was always evaluated over the data range extended by $\pm b_{m}$, and trimmed to size for the purpose of fitting. 

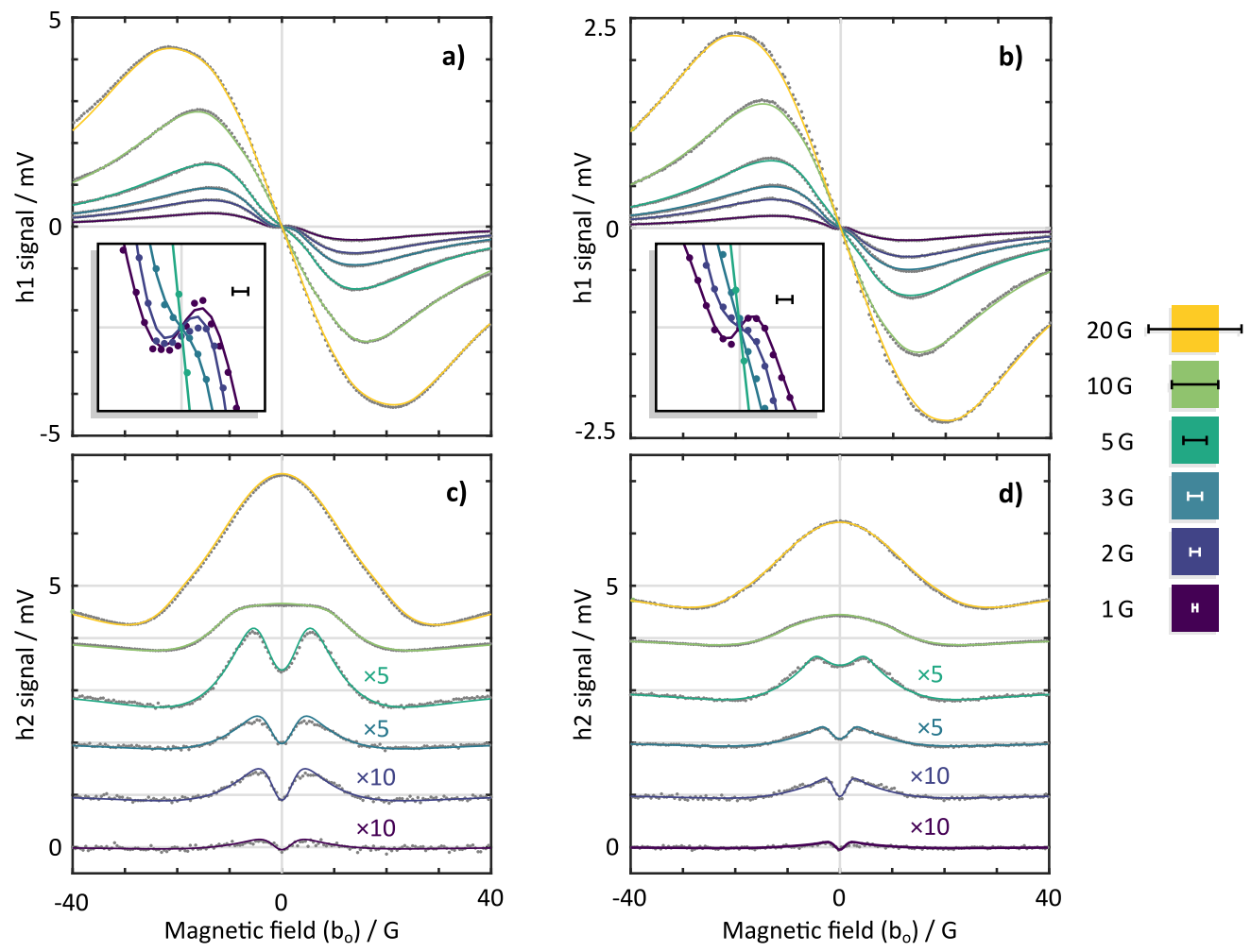

Figure $4.1^{\text {st }}$ and $2^{\text {nd }}$ harmonic data (top and bottom row respectively). All the modulation depths for both harmonics have been fitted simultaneously (see Sec. III B), for PyH10 (left column) and PyD10 (right column). The fits are shown as solid lines coloured by the modulation depth (see legend), while the data is shown as gray points. The scale bars drawn on top of the legend on the right hand side correspond to the scale of the abscissa of all of the plots. The inserts in a) and b) show the detail of the LFE region of the $1^{\text {st }}$ harmonic data. The scale bars in the inserts are $2 \mathrm{G}$ wide. Modulation depths of 10 and $20 \mathrm{G}$ have been omitted from the inserts as they would appear as a featureless vertical line. Note also the change of ordinate scale of b) with respect to a). The $2^{\text {nd }}$ harmonic data in c) and d) has been shifted by progressive integer multiples of $1 \mathrm{mV}$ to allow for easier comparison. Low modulation depths have been scaled up $10 \mathrm{x}$ for 1 and $2 \mathrm{G}$, and $\mathrm{x} 5$ for 3 and $5 \mathrm{G}$.

We have employed a method of bootstrap resampling of the residuals and refitting. ${ }^{31-33}$ This allows the evaluations of "goodness of fit", as well as sampling parameters of interest of the fitted MARY curves. Briefly, the residuals of the best-fit were sampled in blocks, with replacement. The autocorrelation function of the residuals was used to estimate the block size in order to preserve the non-white nature of the new resampled residuals. These were then added onto the best-fit line(s) and refitted. This procedure was repeated 1000 times, and the distribution of the fit values was analysed. The median, lower and upper standard deviation equivalence were extracted, giving the fit parameters $\bar{p}$ and their spread $\delta \bar{p}$. The bootstrapped parameters of interest were the (x,y) position of the MARY curve amplitude at half saturation $\mathrm{B} 1 / 2$, as well as the position of the maximum of the LFE feature.

The fitting was found to take roughly $25 \mathrm{~ms}$ for $\sim 2 \mathrm{k}$ points dataset on a typical desktop PC (Intel(R) Core(TM) i5-6600 CPU @ 3.3 GHz, 8 GB RAM). The subsequent 1 k points bootstrap took correspondingly $\sim 30 \mathrm{~s}$. The majority of this time was spent on the convolution, which averaged $\sim 150 \mu$ s per call with $\sim 2 \times 10^{6}$ calls. This analysis was performed in MATLAB 2017a.

\section{Sample of interest}

The investigated sample is a donor/acceptor, mixture of pyrene (Py) and 1,3-dicyanobenzene (DCB) respectively. ${ }^{34}$ This system has been extensively studied and shows a large magnetic field effect, ${ }^{16,18,35,36}$ as well as a pronounced low field effect ${ }^{14,19}$ making it a perfect model system for this analysis. Briefly, photoexcited $S_{1}$ pyrene forms an exciplex with DCB. The fluorescence of the exciplex is distinctly red shifted compared to that of the ground state. ${ }^{34,37}$ The exciplex can also dissociate into a magnetosensitive radical pair which, due to the high viscosity of the solvent, has a high probability of spin-selective recombination. This means that a large percentage of the exciplex fluorescence is magnetosensitive, leading to large, easily observable MFEs.

Py (98\% Sigma Aldrich 185515) and DCB (98\% Sigma Aldrich 145858) were made up to concentrations of $400 \mu \mathrm{M}$ 


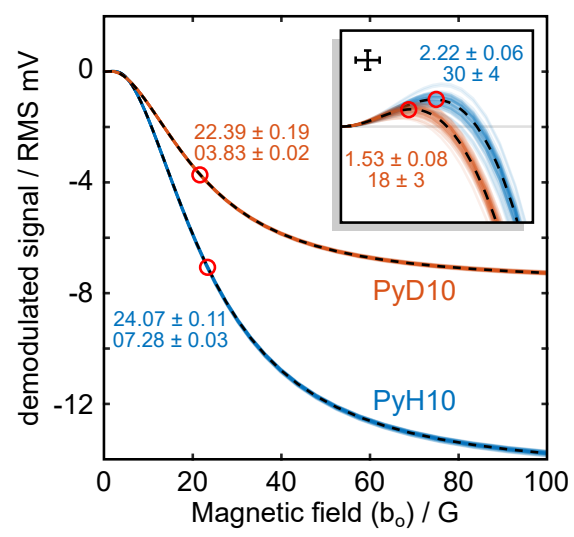

Figure 5. Reconstruction of the MARY curves of PyH10 (blue) and PyD10 (red), based on the fit of the data from Fig. 4. The points of interest, B1/2 and the maximum LFE, are marked, and the bootstrapped values with prediction bounds are given. The insert shows details of the LFE region. The scale cross in the insert is $0.5 \mathrm{G}$ wide and $20 \mu \mathrm{V}$ high.

and $40 \mathrm{mM}$ respectively in pre-prepared 9:1 mixture of cyclohexanol (99\% Sigma Aldrich 105899) and acetonitrile (99.8\% Sigma Aldrich 271004). The concentration of the quencher (DCB) was kept in excess to suppress pyrenepyrene excimer formation. ${ }^{38}$ The solvent mixture was chosen due to its high viscosity, which promotes the formation of magnetosensitive exciplex species. Its high dielectric constant also stabilizes charged species, including the exciplex and the radical pair. ${ }^{15}$

We have measured both natural abundance PyH10 and fully perdeuterated PyDio pyrene samples. Deuteration decreases the molecular hyperfine coupling constant of the pyrenyl radical cation in the radical pair, and therefore should strongly affect the size and shape of the MARY curve. $^{20}$

\section{RESULTS AND DISCUSSION}

We measured both the first $\left(h_{1}\right)$ and second $\left(h_{2}\right)$ harmonic data over a range of modulation depths for both $\mathrm{PyH}_{10} / \mathrm{DCB}$ and PyD10/DCB samples. For each of the two samples, phased signals for all of the harmonics and modulation depths were fitted simultaneously as described in Sec. III B. The results of the fit can be seen in Fig. 4.

It was found that the simulation using $M_{L}$ as a model for the MARY curve can reproduce both the shape and the magnitude of the signal very well. This is promising, especially since the choice to use a difference of Lorentzians to model the LFE is somewhat arbitrary. Special care had to be taken for the signal magnitude to stay constant over the course of the experiment. Any change in the signal magnitude for long experiments has been attributed to 1) sample photodegradation and 2) heating of the modulation coils at very high modulation depths leading to increased impedance.
The sensitive detection of the LFE is one of the main goals of this work. We will therefore discuss a selection of the apparent features of the data that relate specifically to it. The LFE gives rise to a clearly visible feature around $2 \mathrm{G}$ for the harmonic data (see insert of Fig. 4 a,b). As discussed, however, the information content of the $h_{1}\left(\sim 1^{\text {st }}\right.$ derivative) signal in this region drops quickly with increasing modulation depth, and the LFE feature can only be seen clearly for $2 \times b_{m}$ in the range of 1-3 G. Conversely, the $h_{2}$ data shows a discernible LFE feature (dip/inflection in the middle of the signal) all the way up to $2 \times b_{m}=10 \mathrm{G}$ for both datasets. $h_{2}$ data also contains information about the shoulders of the MFE at higher fields, but the signal quickly vanishes at higher fields (compare e.g. signal magnitude for $b_{o}=10 \mathrm{G}$ for low modulation depths). This means that $h 2$ alone is not sufficient for the reconstruction of $M$.

Good estimates of the MARY curve could not be obtained from the $2 \times b_{m}=20 \mathrm{G}$ data. Higher overtones become easier to detect at higher modulation depths since the magnitude of the signal scales approximately with powers of $2 \times b_{m}$ (see Eq. 13). Hence, a good fit at $2 \times b_{m}=20 \mathrm{G}$ could succeed if higher overtone data were measured (at the time this was not possible, see supplementary information) and incorporated into the fit. ${ }^{27}$ Interestingly, the LFE in the $h_{1}$ data for $\mathrm{PyH}_{10}$ gives rise to a small deflection in the gradient of the signal close to peak signal at $-20 \mathrm{G}$ (Fig. 4). This is predicted exactly by the fit and indeed makes perfect sense as that is where the oscillating field begins to "see" the LFE. This apparently visible LFE feature did not, however, improve the quality of the fit of that data alone, as the information about the LFE had been distributed too thinly across the signal.

The analysis of the fitted data suggests that measurements of a magneto-sensitive chemical system at higher modulation depths - where $2 \times b_{m}$ is up to 5 times the expected LFE width, ought to succeed in reconstructing the underlying MARY curve $M$ with the correct LFE feature. This can be done by simultaneous fitting of the $h_{1}$ and $h_{2}$ signals (and higher overtones if signal magnitude is attainable), since the information about the higher order terms in Taylor expansion of the model of $M$ (Fig. 2) is retained by the higher order harmonics. A subset of reconstructed MARY curves $M$ from this analysis, as well as the bootstrapped fits ${ }^{33}$ serving as prediction bounds, can be seen in Fig. 5 . It is clear that the PyD10 sample gives rise to a lower magnitude field effect, with a narrower LFE feature (see insert of Fig. 5). The bootstrapped (x,y) coordinates of $\mathrm{B} 1 / 2$ and the maximum LFE point are shown on the figure. The reported uncertainty is the standard deviation of the bootstrapped heuristics which were found to be approximately normally distributed (this was less of the case for the LFE maximum, as it sits close to the origin, which is a fixed point of $M_{L}$ ). Compared to the natural isotopic abundance PyH10, the LFE feature of the perdeuterated pyrene shrinks and the magnitude of the overall field effect decreases. A strong effect of deuteration on the LFE is expected, since there is approximately 6.5-fold difference in the hyperfine coupling con- 
stants of the deuterons and protons. ${ }^{39}$ The change in hyperfine MFE magnitude is also pronounced due to the resulting change in singlet probability. This can be contrasted to works of Richert et al. ${ }^{40}$, and Rodgers et al. ${ }^{39}$, both of whom showed no significant change in the magnitude of the MFE upon deuteration of pyrene in the pyrene/dimethylalanine system - a behaviour attributed to degenerate electron exchange. ${ }^{19}$ Therefore, the fact that we $d o$ observe such change in our measurements suggests the electron selfexchange does not play a major role in the Py/DCB system, and the majority of the reaction proceeds through geminate exciplexes.

Although the main result of this work is time-independent (Eq. 36), its derivation assumes fast modulation (the assumption of $\eta \gg 1$ ) and, effectively an oscillatory steadystate of the sample of interest. Hence, although the broadening and harmonic distortion effects are frequency independent, the magnitude of samples' response (in this case the field effect) might be. In the case of the chemical response of the sample, as is the case in this work, these are the reaction rates of the individual reaction steps leading to, as well as downstream of, the magnetosensitive step. ${ }^{41,42}$ To perform such an experiment on the ModMARY apparatus, the signal of the sample of interest would have to be acquired at a range of modulation frequencies $\left(f_{m}\right)$ straddling the timescale of the slow MFE component. The prompt MFE is then expected to contribute to the signal at all $f_{m}$, while the response the slow component will be suppressed at high $f_{m}$, leading to a change in the observed signal magnitude.

It is important to note that the technique described here is model-based - the results of a fit are based upon an assumption that the underlying model of $M$ is correct. For an unknown dataset this may not necessarily be the case and the "best" model has to be chosen out of a few proposed. There are many ways of approaching such model selection problems. One way is to consider the degree to which the information contained in the data is expressed by each of the models, while penalising models with many parameters in order to avoid overparametrisaton. ${ }^{43,44}$ Another approach is to compete the models against one-another pairwise, and test the null hypothesis that the models approximate the data (more accurately the data generating process) equally well against the alternate hypothesis that one approximates the data better than another. ${ }^{45}$ In simple cases, and when the model has far fewer degrees of freedom than the data, determination of the best model can be made by a comparison of performance heuristics, e.g. root-meansquare residuals, variance of bootstrapped fit parameters $\bar{p}$ or cross-validated in-sample prediction power (see supplementary information). For the purposes of MFE research, as a first-order approximation, we suggest the $M_{L}$ function described above as a simple model of a $M$. Better, fast and more insightful models of a MARY curve are being actively developed. ${ }^{9}$ The technique described here can, however, be used to model most stimulus-modulated derivative signals (those which conform with the assumptions made in the derivation). The correctness of $M$ model must, in that case, be carefully considered.

\section{CONCLUSIONS}

With minimal assumptions, we have shown that the stimulus modulation broadening can be represented as a convolution, and derived the exact shape of the underlying convolution kernel. Additionally, our derivation has captured the effects of harmonic distortion and generation of overtones of the modulated signal. Hence, we have introduced a model-based technique to model an arbitrary stimulusmodulated derivative signal, and its overtones. We have shown its utility with specific application to modelling of Modulated MARY spectroscopy data of magnetic field effects. We were able to resolve a sub-Gauss shift in the lowfield effect feature upon deuteration, which is of great interest for the field of RP-based MFEs ${ }^{46,47}$ and magnetoreception research. ${ }^{7,48}$

Multiple harmonics of the same sample response can be detected simultaneously with multiple quadrature detectors or a single multichannel one, especially if digital lockin techniques are employed. We therefore propose that the technique of multi-harmonic detection and reconstruction described in this work is applicable, not only in Modulated MARY spectroscopy, but in a wide range of techniques which make use of stimulus modulation.

\section{CONFLICTS OF INTEREST}

The authors declare no conflicts of interest.

\section{DATA AVAILABILITY}

The data shown, and the analysis scripts demonstrating the methodology outlined, have been deposited in the Oxford University Research Archive (ORA) at ora .ox .ac .uk. ${ }^{1}$

\section{AUTHOR CONTRIBUTIONS}

O.F.V.E. performed the experiments. J.G.S. developed the initial concept and numerical simulations. M.K. built the experiment, performed the analysis and wrote the manuscript. All authors discussed the results and commented on the manuscript.

\section{ACKNOWLEDGEMENTS}

First and foremost, the authors would like to thank Prof. Christiane Timmel for supervision of their studies and insightful comments on the manuscript. We would like to thank the EMF Biological Research Trust and Air Force Office of Scientific Research (AFOSR, USAF award number 
FA9550-14-1-0095) for funding; Electronics Workshop in Physical and Theoretical Chemistry Laboratory (University of Oxford), and especially Neville Baker, Phillip Hurst, John Adams and Timothy Powell for building components of the experiment; Dr. Christan Kerpal, Gabriel Moise, Matthew Golesworthy and Victoire Déjean for proofreading; Dr. Sabine Richert for helpful discussions and Dr. William Myers for paper suggestions. Further, the authors would like to extend their thanks to The On-Line Encyclopaedia of Integer Sequences, ${ }^{49}$ and the Matplotlib development team. ${ }^{50}$

\section{GLOSSARY}

For the benefit of the reader, we provide the following glossary of some of the more context-specific symbols used throughout the text (especially in Sec. II)

$f_{m}$ Frequency of the oscillating component of the modulated stimulus (magnetic field).

$b_{m}$ Amplitude of the oscillating component of the modulated stimulus.

$b_{o}$ Static (offset) component of the magnetic field.

$b$ Total applied magnetic field - sum of the offset and modulated components (Eq. 9).

$\tau$ Time constant of the exponential window of the LIA.

$I$ Input to the LIA. This is the result of the modulated stimulus (magnetic field, $b$ ) on the transfer function ( $M$, MARY curve, Fig. $1 b)$.

$R$ Reference oscillation of the LIA - in-phase harmonic of the modulated stimulus (Eq. 2).

$h$ Integer harmonic constant. Reference oscillation is an $h^{\text {th }}$ harmonic of the modulation frequency.

$\eta$ Number of radians of oscillation of the reference frequency $(R)$ within a single time constant $(\tau)$ of the LIA.

$D_{h}$ Time-domain detection kernel of the LIA (Eq. 4), corresponding to the harmonic constant $h$.

$S$ Output of the LIA - demodulated, and LP-filtered input (I).

$M$ Transfer function (MARY curve) from the stimulus (magnetic field, $b$ ) to the samples' response (Fig. 1b). Derivatives of the transfer function with respect to the stimulus are denoted with'.

$\mathfrak{t}$ Time, counted in number of oscillation periods of the modulated stimulus (Eq. 16)

$j$ Period counter for the integral split (Eq. 16).

$J$ Average height of the exponential window component of the detection kernel $(D)$ within the $j^{\text {th }}$ period of the modulated stimulus (Eq. 18).

$E$ Shape of the exponential window of the detection kernel within the any one period of the modulated stimulus (Eq. 19).

$\mathfrak{m}$ Modulation parameter-oscillating part of the stimulus, scaled to unit amplitude (Eq. 24).

$\mathcal{T}_{n} n^{\text {th }}$ Chebyshev polynomial of the $1^{\text {st }}$ kind (Eq. 29).

$\mathcal{U}_{n} n^{\text {th }}$ Chebyshev polynomial of the $2^{\text {nd }}$ kind (Eq. 31).

$K_{h}$ Convolution kernel which models the $h^{\text {th }}$ harmonic of the action of the stimulated modulus on the derivative of the transfer function $\left(M^{\prime}\right.$, Eq. 36 and 37$)$.
$M_{L}$ Double Lorentzian model of the MARY curve (Eq. 39).

\section{REFERENCES}

${ }^{1}$ Olivia Foster Vander Elst, Marcin Konowalczyk, and Jonathan Storey. Convolution-based modelling of the stimulus modulation broadening in derivative spectroscopy, 2020. https://www.doi.org/10.5287/ bodleian: OvD9azdXR.

${ }^{2}$ Christian Kerpal, Sabine Richert, Jonathan G. Storey, Smitha Pillai, Paul A. Liddell, Devens Gust, Stuart R. Mackenzie, P. J. Hore, and Christiane R. Timmel. Chemical compass behaviour at microtesla magnetic fields strengthens the radical pair hypothesis of avian magnetoreception. $\mathrm{Na}$ ture Communications, 10(1):1-7, 2019.

${ }^{3}$ Kiminori Maeda, Kevin B. Henbest, Filippo Cintolesi, Ilya Kuprov, Christopher T. Rodgers, Paul A. Liddell, Devens Gust, Christiane R. Timmel, and Peter J. Hore. Chemical compass model of avian magnetoreception. Nature, 453(7193):387-390, 2008.

${ }^{4}$ Charles B. Grissom. Magnetic field effects in biology: A survey of possible mechanisms with emphasis on radical-pair recombination. Chemical Reviews, 95(1):3-24, 1995.

${ }^{5}$ Christopher T. Rodgers and Peter J. Hore. Chemical magnetoreception in birds: The radical pair mechanism. Proceedings of the National Academy of Sciences of the United States of America, 106(2):353-360, 2009.

${ }^{6}$ Vladimir N. Binhi and Frank S. Prato. Rotations of macromolecules affect nonspecific biological responses to magnetic fields. Scientific Reports, 8(1):1-11, 2018.

${ }^{7}$ Peter J. Hore and Henrik Mouritsen. The radical-pair mechanism of magnetoreception. Annual Review of Biophysics, 45(1):299-344, 2016.

${ }^{8}$ Christiane R. Timmel, U. Till, Brian Brocklehurst, K. A. Mclauchlan, and Peter J. Hore. Effects of weak magnetic fields on free radical recombination reactions. Molecular Physics, 95(1):71-89, 1998.

${ }^{9}$ Alan M. Lewis, Thomas P. Fay, David E. Manolopoulos, Christian Kerpal, Sabine Richert, and Christiane R. Timmel. On the low magnetic field effect in radical pair reactions. Journal of Chemical Physics, 149(3):034103, 2018.

${ }^{10}$ M. L. Meade. Advances in lock-in amplifiers. Journal of Physics E: Scientific Instruments, 15:395-403, 1982.

${ }^{11}$ Principles of lock-in detection and the state of the art zürich instruments. Technical report, Zürich Instruments, 2016. www. zhinst. com.

${ }^{12}$ Low level optical detection using lock-in amplifier techniques. Technical report, AMTEK Advanced Measurment Technology Inc., 2008.

${ }^{13}$ About lock-in amplifiers. Technical report, Stanford Research Systems Inc., 2011. www. thinkSRS. com.

${ }^{14}$ C.A. Hamilton, J.P. Hewitt, K.A. McLauchlan, and U.E. Steiner. High resolution studies of the effects of magnetic fields on chemical reactions. Molecular Physics, 65(2):423-438, 1988.

${ }^{15}$ S. N. Batchelor, C. W. M. Kay, K. A. Mclauchlan, and I. A. Shkrob. Timeresolved and modulation methods in the study of the effects of magneticfields on the yields of free-radical reactions. Journal of Physical Chemistry, pages 13250-13258, 1993.

${ }^{16}$ Christopher J. Wedge. Radiofrequency Magnetic Field Effects on Radical Pair Recombination Reactions. D.phil, University of Oxford, 2009.

${ }^{17}$ Kunal Pal, Daniel R. Kattnig, Günter Grampp, and Stephan Landgraf. Experimental observation of preferential solvation on a radical ion pair using mary spectroscopy. Physical Chemistry Chemical Physics, 14(9):31553161, mar 2012.

${ }^{18}$ Kelly-Anne Ferguson. An Investigation of isotropic and anisotropic magnetic field effects in fluorescent systems. $\mathrm{PhD}$ thesis, University of Oxford, 2013. https://ora.ox.ac.uk/objects/ora: 8978.

${ }^{19}$ M. Justinek, G. Grampp, S. Landgraf, Peter J. Hore, and N. N. Lukzen. Electron self-exchange kinetics determined by mary spectroscopy: theory and experiment. Journal of the American Chemical Society, 126(17):56355646, may 2004.

${ }^{20}$ Christiane R. Timmel and Kevin B. Henbest. A study of spin chemistry in weak magnetic fields. Philosophical Transactions of the Royal Society A, 362(1825):2573-2589, dec 2004.

${ }^{21}$ Nan Yang and Adam E. Cohen. Optical imaging through scattering media via magnetically modulated fluorescence. Optical Express, 18(25):2546125467, 2010. 
${ }^{22}$ Joshua P. Beardmore, Lewis M. Antill, and Jonathan R. Woodward. Optical absorption and magnetic field effect based imaging of transient radicals. Angewandte Chemie International Edition, 54(29):8494-8497, 2015.

${ }^{23}$ Lewis M. Antill, Joshua P. Beardmore, and Jonathan R. Woodward. Time-resolved optical absorption microspectroscopy of magnetic field sensitive flavin photochemistry. Review of Scientific Instruments, 89(2):023707, 2018.

${ }^{24}$ Paul Horowitz and Winfield Hill. The Art Of Electronics. Cambridge University Press, $3^{\text {rd }}$ edition, 2015. https: //artofelectronics. net/.

${ }^{25}$ S. E. Tavares. A comparison of integration and low-pass filtering. IEEE Transactions on Instrumentation and Measurement, 15:33-38, 1966.

${ }^{26}$ Peter J. Hore, Jonathan Jones, and Stephen Wimperis. NMR: The Toolkit. Oxford University Press, $2^{\text {nd }}$ edition, 2015.

${ }^{27}$ Mark Tseitlin, Sandra S. Eaton, and Gareth R. Eaton. Reconstruction of the first-derivative epr spectrum from multiple harmonics of the fieldmodulated continuous wave signal. Journal of Magnetic Resonance, 209(2):277-281, 2011.

${ }^{28}$ National Instruments. Extract single tone information vi. Part of the Signal Processing Toolbox, LabVIEW 2015, Online documentation accessed November 2019, https://zone.ni.com/reference/ en-XX/help/371361N-01/lvwave/extract_single_tone_info/.

${ }^{29}$ J. G. Ziegler and N. B. Nichols. Optimum settings for automatic controllers. InTech, 42(6):94-100, 1995.

${ }^{30}$ J. A. Nelder and R. Mead. A simplex method for function minimization. The Computer Journal, 7(4):308-313, 1965.

${ }^{31}$ Philip. M. Dixon. Bootstrap resampling. Encyclopedia of Environmetrics, 2006.

${ }^{32}$ James Carpenter and John Bithell. Bootstrap confidence intervals: when, which, what? a practical guide for medical statisticians. Statistics in Medicine, 19:1141-1164, 2000.

${ }^{33}$ S. Huet, A. Bouvier, M.-A. Poursat, and E. Jolivet. Statistical Tools for Nonlinear Regression. Springer US, $2^{\text {nd }}$ edition, 2010.

${ }^{34}$ Susan L. Mattes and Samir Farid. Exciplexes and electron transfer reactions. Science, 226(4677):917-921, 1984.

${ }^{35}$ Günter Grampp, M. Justinek, and Stephan Landgraf. Magnetic field effects on the pyrene-dicyanobenzene system: determination of electron self-exchange rates by mary spectroscopy. Molecular Physics, 100(8):1063-1070, apr 2002.

${ }^{36}$ C. J. Wedge, Christopher T. Rodgers, Stuart A. Norman, Neville Baker, Kiminori Maeda, Kevin B. Henbest, C. R. Timmel, and P. J. Hore. Radiofrequency polarization effects in low-field electron paramagnetic resonance. Physical Chemistry Chemical Physics, 11(31):6573-6579, 2009.

${ }^{37}$ Daniel R. Kattnig, Arnulf Rosspeintner, and Günter Grampp. Magnetic field effects on exciplex-forming systems: the effect on the locally ex- cited fluorophore and its dependence on free energy. Physical Chemistry Chemical Physics, 13(8):3446-3460, feb 2011.

${ }^{38}$ M. E. Sigman, P. F. Schuler, M. M. Ghosh, and R. T. Dabestani. Mechanism of pyrene photochemical oxidation in aqueous and surfactant solutions. Environmental Science \& Technology, 32(24):3980-3985, 1998.

${ }^{39}$ Christopher T. Rodgers, Stuart A. Norman, Kevin B. Henbest, Christiane R. Timmel, and P. J. Hore. Determination of radical re-encounter probability distributions from magnetic field effects on reaction yields. Journal of the American Chemical Society, 129(21):6746-6755, 2007.

${ }^{40}$ Sabine Richert, Arnulf Rosspeintner, Stephan Landgraf, Günter Grampp, Eric Vauthey, and Daniel R. Kattnig. Time-resolved magnetic field effects distinguish loose ion pairs from exciplexes. Journal of the American Chemical Society, 135(40):15144-15152, oct 2013.

${ }^{41}$ Daniel R. Kattnig, Emrys W. Evans, Victoire Déjean, Charlotte A. Dodson, Mark I. Wallace, Stuart R. Mackenzie, Christiane R. Timmel, and P. J. Hore. Chemical amplification of magnetic field effects relevant to avian magnetoreception. Nature Chemistry, 8(4):384-391, 2016.

${ }^{42}$ Victoire Déjean, Marcin Konowalczyk, Jamie Gravell, Matthew J. Golesworthy, Catlin Gunn, Nils Pompe, Olivia Foster Vander Elst, Ke-Jie Tan, Mark Oxborrow, Dirk G. A. L. Aarts, Stuart R. Mackenzie, and Christiane R. Timmel. Detection of magnetic field effects by confocal microscopy. Chemical Science, 11:7772-7781, 2020.

${ }^{43} \mathrm{M}$. R. Forster. Model selection in science: the problem of language variance. The British Journal for the Philosophy of Science, 50(1):83-102, 03 1999.

${ }^{44}$ Jürgen Mayer, Khaled Khairy, and Jonathon Howard. Drawing an elephant with four complex parameters. American Journal of Physics, 78(6):648-649, 2010.

${ }^{45}$ Quang H. Vuong. Likelihood ratio tests for model selection and nonnested hypotheses. Econometrica, 57(2):307-333, 1989.

${ }^{46}$ Jukka Juutilainen, Mikko Herrala, Jukka Luukkonen, Jonne Naarala, and P. J. Hore. Magnetocarcinogenesis: Is there a mechanism for carcinogenic effects of weak magnetic fields? Proceedings of the Royal Society $B$, 285(1879):20180590, 2018.

${ }^{47}$ Chris Sampson, Robert H. Keens, and Daniel R. Kattnig. On the magnetosensitivity of lipid peroxidation: Two-: versus three-radical dynamics. Physical Chemistry Chemical Physics, 21(25):13526-13538, 2019.

${ }^{48}$ Connie X. Wang, Isaac A. Hilburn, Daw An Wu, Yuki Mizuhara, Christopher P. Cousté, Jacob N.H. Abrahams, Sam E. Bernstein, Ayumu Matani, Shinsuke Shimojo, and Joseph L. Kirschvink. Transduction of the geomagnetic field as evidenced from alpha-band activity in the human brain. eNeuro, 6(2), 2019.

${ }^{49}$ OEIS Foundation Inc. The on-line encyclopedia of integer sequences, 2019. http://oeis.org/A001787.

${ }^{50}$ Choosing colormaps in matplotlib. https://matplotlib.org/3.1.0/ tutorials/colors/colormaps.html. 